UDC 547.728.1

\title{
The synthesis of 5-hetarylamino-3-aryl-1H-indazoles as inhibitors of protein kinase CK2
}

\author{
M. V. Protopopov¹, V. S. Vdovin¹, S. S. Lukashov¹, O. V. Ostrynska1, I. P. Borysenko ${ }^{1,2}$, \\ O. V. Borovykov ${ }^{1}$, S. A. Starosyla ${ }^{1}$, Y. V. Bilokin ${ }^{3}$, O. P. Kukharenko ${ }^{1}$, V. G. Bdzhola ${ }^{1}$, \\ S. M. Yarmoluk ${ }^{1}$ \\ ${ }^{1}$ Institute of Molecular Biology and Genetics, NAS of Ukraine \\ 150, Akademika Zabolotnoho Str., Kyiv, Ukraine, 03143. \\ ${ }^{2}$ LLC "Scientific and service firm "Otava" \\ 150, Akademika Zabolotnoho Str., Kyiv, Ukraine, 03143 \\ ${ }^{3}$ OTAVA Ltd \\ 400 Applewood Crescent, Unit 100, Vaughan, Ontario, L4K 0C3 Canada \\ mykola.protopopov@imbg.org.ua.
}

\begin{abstract}
Aim. Basing on our earlier finding of inhibitory activity of 5-(4-quinazolylamino)-3-arylindazoles against human protein kinase CK2, the synthesis of new nitrogen containing heterocyclic derivatives was performed in order to find novel inhibitors of this kinase. Methods. Organic synthesis, NMR spectroscopy. Results. A series of 4-chloroquinazolines, 4-chloroquinolines, 4-chloropyrazolo[3,4-d]pyrimidines and 4-chlorothieno[2,3-d]pyrimidine was synthesized. Reaction of these intermediates with 5-amino-3-(3,4-dichlorophenyl)-indazole gave us a series of 14 novel heterocyclic derivatives of 5-amino-3-arylindazole. Conclusions. Besides new quinazoline derivatives - the quinoline and thieno[2,3-d]pyrimidine derivatives of similar structure but different polarity were obtained. Also a series of 1-methylpyrazolo[3,4-d]pyrimidine derivatives with decreased lipophilicity was synthesized.
\end{abstract}

K e y w o r d s: synthesis, indazole, quinazoline, thieno[2,3-d]pyrimidine, pyrazolo[3,4-d]pyrimidine, protein kinase $\mathrm{CK} 2$ inhibitor

\section{Introduction}

Serine/threonine protein kinase CK2 is one of the first discovered kinases [1]. Despite the long history of CK2 investigations, a number of its functions are not completely determined. This is due to CK2 unique properties, such as constitutive activity, high pleiotropicity (CK2 phosphorylates more than 500 proteins) [2], dual-substrate specificity [3,4], and localization in different cell compartments [5]. Experimental results clearly showed that $\mathrm{CK} 2$ is involved in

(C) 2020 M. V. Protopopov et al.; Published by the Institute of Molecular Biology and Genetics, NAS of Ukraine on behalf of Biopolymers and Cell. This is an Open Access article distributed under the terms of the Creative Commons Attribution License (http://creativecommons.org/licenses/by/4.0/), which permits unrestricted reuse, distribution, and reproduction in any medium, provided the original work is properly cited 
regulating transcription, translation, cell proliferation, survival, and apoptosis [6]. Overexpression and overactivity of CK2 are associated with the development of neurodegenerative, inflammatory, cardio-vascular diseases, virus infections, and all the cancers that have been examined [7,8]. Therefore, smallmolecular inhibitors of CK2 would be important compounds for the development of clinical agents.

The search for CK2 inhibitors has been carried out for over 20 years [9, 10]. Most of the identified CK2 inhibitors belong to coumarins [11], halogenated benzimidazoles (TBB) [12], anthraquinones (emodin) [13], indoloquinazolines (IQA) [14], thieno[2,3-d] pyrimidines $[15,16], 4,5,6,7$-tetrahalogeno- $1 \mathrm{H}$-isoindole-1,3(2H)-diones [17], flavones derivatives (luteolin, FLC26, FNH79) [18-20], 1,3-thiazole-5-carboxylic acid derivatives [21], quinolones [22], aurones [23, 24] and naphthyridines (CX-4945, CX-5011) [25, 26].

Indazole, also called benzopyrazole, is a heterocyclic molecule in which the pyrazole ring is fused with the benzene ring. Indazole derivatives have been reported as inhibitors of FGFR1 kinase, Aurora kinase, Pim kinase, Bcr-Abl tyrosine kinase [27]. Also, previously it has been described that 5-amino-3-arylindazole core (Fig. 1) could be a good starting point for the protein kinase inhibitors design [28].

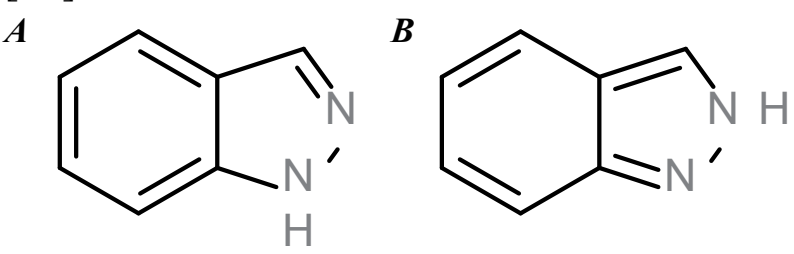

Fig. 1. Chemical structure of indazole $1 \mathrm{H}$-tautomer $(\boldsymbol{A})$ and $2 \mathrm{H}$-tautomer $(\boldsymbol{B})$.
Taking into account this fact, the main aim of this work was to find novel inhibitors of human protein kinase CK2 among the 5-hetarylamino- $1 H$-indazole derivatives.

\section{Materials and Methods}

Starting materials and solvents were purchased from commercial suppliers and used without further purification. ${ }^{1} \mathrm{H}$ NMR spectra were recorded on a Varian Mercury 300 instrument at $302 \mathrm{MHz}$ or Varian VXR 400 instrument at $400 \mathrm{MHz} .{ }^{13} \mathrm{C}$ NMR spectra were recorded on a Varian Mercury 300 instrument at $76 \mathrm{MHz}$ or Varian VXR 400 instrument at $101 \mathrm{MHz}$. Chemical shifts were described as parts per million $(\delta)$ downfield from an internal standard of tetramethylsilane. All tested compounds had $\geq 95 \%$ purity as determined by this method.

(2 - Chloro-5-nitrophenyl)-3,4dichlorobenzophenone, 5. To a stirred suspension of $25 \mathrm{~g}(0.124 \mathrm{~mol})$ of 2-chloro-5-nitrobenzoic acid 4 in $50 \mathrm{ml}$ of 1,2-dichloroethane, 2-3 drops of DMF and $15 \mathrm{ml}(0.205 \mathrm{~mol})$ of thionyl chloride were added. The mixture was being refluxed until the complete dissolution of acid and cessation of release of gaseous $\mathrm{SO}_{2}$ and hydrogen chloride in a bubble counter over the condenser. Then volatile components were evaporated under reduced pressure, $25 \mathrm{ml}$ of 1,2-dichloroethane were added to the residue and evaporation of volatile components was repeated. To the crude 2-chloro-5-nitrobenzoyl chloride, $70 \mathrm{ml}(0.62 \mathrm{~mol})$ of 1,2-dichlorobenzene and $17 \mathrm{~g}(0.127 \mathrm{~mol})$ of aluminium chloride were added. The mixture was being heated at $100{ }^{\circ} \mathrm{C}$ under stirring for 4-6 hours until cessation of release of gaseous hydrogen chloride. After cooling to room temperature, the viscous reaction mixture was diluted with 
$250 \mathrm{ml}$ of cold water and $10 \mathrm{ml}$ of conc. hydrochloric acid. Crude reaction product was extracted with $100 \mathrm{ml}$ of $\mathrm{CH}_{2} \mathrm{Cl}_{2}$. The organic layer was evaporated at $100{ }^{\circ} \mathrm{C}$ under $20 \mathrm{~mm}$ $\mathrm{Hg}$. The residue was recrystallized from isopropanol giving $35 \mathrm{~g}(85 \%)$ of 5 as yellow crystals. ${ }^{1} \mathrm{H}$ NMR $\left(400 \mathrm{MHz}, \mathrm{DMSO}-d_{6}\right)$ : $\delta$ $8.4(\mathrm{dd}, \mathrm{J}=8.8 \mathrm{~Hz}, 2.7 \mathrm{~Hz}, 1 \mathrm{H}), 8.38(\mathrm{~d}, \mathrm{~J}=$ $2.7 \mathrm{~Hz}, 1 \mathrm{H}), 7.92(\mathrm{~d}, \mathrm{~J}=2.0 \mathrm{~Hz}, 1 \mathrm{H}), 7.89(\mathrm{~d}$, $\mathrm{J}=8.8 \mathrm{~Hz}, 1 \mathrm{H}), 7.75(\mathrm{~d}, 8.5 \mathrm{~Hz}, 1 \mathrm{H}), 7.7(\mathrm{dd}$, $\mathrm{J}=8.5 \mathrm{~Hz}, 2.0 \mathrm{~Hz}, 1 \mathrm{H})$.

3-(3,4-Dichlorophenyl)-1H-indazol-5amine, 6. To a solution of $35 \mathrm{~g}(0.106 \mathrm{~mol})$ of ketone 5 in $50 \mathrm{ml}$ of dimethylformamide, $7 \mathrm{ml}$ of hydrazine hydrate were added. The reaction mixture was being refluxed for 1 hour. After cooling to room temperature 5-nitro-3-(3,4dichlorophenyl)- $1 H$-indazole hydrochloride appeared as a bright yellow precipitate. It was separated by filtration and further recrystallized from dimethylformamide to remove the impurity of (2,3-dichlorophenyl)- $1 H$-indazole isomer. After recrystallization, $8.5 \mathrm{~g}(23 \%)$ of pure 5-nitro-3-(3,4-dichlorophenyl)- $1 H$-indazole hydrochloride were obtained as a yellow powder. ${ }^{1} \mathrm{H}$ NMR $\left(302 \mathrm{MHz}, \mathrm{DMSO}-d_{6}\right) \delta$ 13.92 (br s, 1H), $8.91(\mathrm{~d}, J=2.1 \mathrm{~Hz}, 2 \mathrm{H}), 8.24$ $(\mathrm{dd}, J=9.2,2.0 \mathrm{~Hz}, 2 \mathrm{H}), 8.15(\mathrm{~d}, J=2.0 \mathrm{~Hz}$, $2 \mathrm{H}), 7.99(\mathrm{dd}, J=8.4,2.0 \mathrm{~Hz}, 2 \mathrm{H}), 7.76(\mathrm{~d}$, $J=9.2 \mathrm{~Hz}, 2 \mathrm{H}), 7.74(\mathrm{~d}, J=8.4 \mathrm{~Hz}, 2 \mathrm{H}) ;{ }^{13} \mathrm{C}$ NMR (76 MHz, DMSO- $\left.d_{6}\right) \delta 144.1,143.7$, 142.5, 133.0, 132.2, 131.6, 131.5, 128.5, 127.3, $121.5,119.1,118.6,112.0$.

$8.5 \mathrm{~g}$ of 5-nitro-3-(3,4-dichlorophenyl)- $1 \mathrm{H}$ indazole hydrochloride were dissolved in a mixture of $300 \mathrm{ml}$ of isopropanol and $100 \mathrm{ml}$ dimethylformamide, then $3 \mathrm{~g}$ of Raney nickel were added. Well stirred reaction mixture was heated under reflux while $10 \mathrm{ml}$ of hydrazine hydrate were being added slowly dropwise. The reduction process took at least 8 hours and an additional amount of hydrazine hydrate was added if disappearance of deep yellow-orange coloration of reaction mixture did not take place. Hot reaction mixture was filtered from nickel and solvents were evaporated under reduced pressure. Solid residue was washed with water and dried giving $5.35 \mathrm{~g}(78 \%)$ of 6 as red-brown solid. ${ }^{1} \mathrm{H}$ NMR $(302 \mathrm{MHz}$, DMSO- $\left.d_{6}\right) \delta 13.04($ br s, $1 \mathrm{H}), 8.05(\mathrm{~d}, J=$ $1.5 \mathrm{~Hz}, 1 \mathrm{H}), 7.86\left(\mathrm{dd}, \mathrm{J}_{1}=9.0 \mathrm{~Hz}, \mathrm{~J}_{2}=1.5 \mathrm{~Hz}\right.$, $1 \mathrm{H}), 7.71(\mathrm{~d}, J=8.4 \mathrm{~Hz}, 1 \mathrm{H}), 7.32(\mathrm{~d}, J=$ $8.8 \mathrm{~Hz}, 1 \mathrm{H}), 7.09(\mathrm{~s}, 1 \mathrm{H}), 6.86\left(\mathrm{dd}, \mathrm{J}_{1}=8.8 \mathrm{~Hz}\right.$, $\left.\mathrm{J}_{2}=1.6 \mathrm{~Hz}, 1 \mathrm{H}\right), 5.01(\mathrm{~s}, 2 \mathrm{H}) ;{ }^{13} \mathrm{C} \mathrm{NMR}$ $\left(76 \mathrm{MHz}, \mathrm{DMSO}-d_{6}\right) \delta 144.3,138.8,136.6$, $135.8,131.9,129.5,127.6,121.5,118.7,111.6$, 100.2 .

General procedure of synthesis of heterocyclic chloro derivatives. To a suspension of $20 \mathrm{mmol}$ of heterocyclic compound 8-21 in $20 \mathrm{ml}$ of phosphorous oxychloride[,] $4.2 \mathrm{~g}$ (20 mmol) of phosphorous pentachloride were added. The mixture was being refluxed under stirring until complete dissolution of starting material. After cooling to room temperature the volatile material was evaporated under reduced pressure. Residue was dissolved in 50 $\mathrm{ml}$ of chloroform and carefully basified with $\mathrm{NaHCO}_{3}$ solution under good stirring (caution! effervescence takes place). Organic layer was separated, dried with $\mathrm{Na}_{2} \mathrm{SO}_{4}$ and evaporated, leaving chloro-derivative $\mathbf{2 2 - 3 5}$, that was immediately used in the next step without purification.

General procedure of arylation of 5-amino-3-(3,4-dichlorophenyl)-1H-indazol 6. A solution of $2.78 \mathrm{~g}(10 \mathrm{mmol})$ of 6 in $5 \mathrm{ml}$ of dimethylformamide was added to the $10 \mathrm{mmol}$ 
of corresponding chloro-derivative 22-35. The mixture was refluxed for 3-5 min to complete dissolution of all components and cooled to room temperature. Solid hydrochloride salts of products 36-49 were filtered off, washed with dimethylformamide and acetone. Soluble in DMF products were converted to the bases by adding $0.25 \mathrm{ml}$ of triethylamine and $25 \mathrm{ml}$ of water to the reaction mixture before filtration. Several products were additionally purified with column chromatography on silica gel, using the eluent system $\mathrm{CH}_{2} \mathrm{Cl}_{2}: \mathrm{MeOH}$ 95:5 9:1.

$N$-[3-(3,4-Dichlorophenyl)-1H-indazol-5yl]-quinazoline-4-amine hydrochloride 36. Yield $53 \%$. ${ }^{1} \mathrm{H}$ NMR (400 MHz, DMSO- $\left.d_{6}\right)$ : $\delta 13.45($ br s, $1 \mathrm{H}), 11.2($ br s, $1 \mathrm{H}), 8.86(\mathrm{~d}, 1 \mathrm{H}$, $7.6 \mathrm{~Hz}), 8.73(\mathrm{~s}, 1 \mathrm{H}), 8.43(\mathrm{~s}, 1 \mathrm{H}), 8.14(\mathrm{~s}$, 1H), $7.75(\mathrm{~m}, 2 \mathrm{H}), 7.67(\mathrm{~m}, 2 \mathrm{H}) .{ }^{13} \mathrm{C} \mathrm{NMR}$ $\left(101 \mathrm{MHz}, \mathrm{DMSO}-d_{6}\right) \delta 158.4,156.3,150.5$ $147.3,137.7,137.2,135.9,134.0,132.6,132.9$, $131.4,130.4,129.5,129.5,129.1,128.4,127.5$, 119.0, 117.9, 116.6, 99.5.

$\mathrm{N}$-[3-(3,4-Dichlorophenyl)-1H-indazol-5yl]-2-isopropylquinazolin-4-amine hydrochloride 37. Yield $22 \%$. ${ }^{1} \mathrm{H}$ NMR $(400 \mathrm{MHz}$, DMSO- $\left.d_{6}\right) \delta 13.45$ (br s, $\left.1 \mathrm{H}\right), 9.92($ br s, $1 \mathrm{H})$, $8.92(\mathrm{~s}, 1 \mathrm{H}), 8.58(\mathrm{~d}, J=5.5 \mathrm{~Hz}, 1 \mathrm{H}), 8.14(\mathrm{~s}$, $1 \mathrm{H}), 7.99$ (d, $J=8.2 \mathrm{~Hz}, 1 \mathrm{H}), 7.89(\mathrm{~d}, J=8.5$ $\mathrm{Hz}, 1 \mathrm{H}), 7.79(\mathrm{~d}, J=8.1 \mathrm{~Hz}, 1 \mathrm{H}), 7.74(\mathrm{~d}, J=$ $5.3 \mathrm{~Hz}, 2 \mathrm{H}), 7.64(\mathrm{~d}, J=8.2 \mathrm{~Hz}, 1 \mathrm{H}), 7.57(\mathrm{t}$, $J=5.7 \mathrm{~Hz}, 1 \mathrm{H}), 3.04(\mathrm{p}, J=5.8 \mathrm{~Hz}, 1 \mathrm{H}), 1.28$ $(\mathrm{d}, J=5.9 \mathrm{~Hz}, 6 \mathrm{H}) .{ }^{13} \mathrm{C}$ NMR $(101 \mathrm{MHz}$, DMSO- $\left.d_{6}\right) \delta 169.8,157.8,140.5,138.6,134.5$, 133.7, 132.9, 131.7, 131.0, 129.9, 128.8, 128.1, 127.7, 126.5, 125.4, 122.9, 122.8, 119.7, 113.6, 111.7, 110.6, 37.1, 21.6.

$N$-[3-(3,4-Dichlorophenyl)-1H-indazol-5yl]-2-phenylquinazolin-4-amine hydrochloride
38. Yield $54 \%$. ${ }^{1} \mathrm{H}$ NMR ( $302 \mathrm{MHz}$, DMSO- $d_{6}$ ) $\delta 13.54($ br s, $1 \mathrm{H}), 10.06($ br s, $1 \mathrm{H}), 8.76(\mathrm{~s}$, $1 \mathrm{H}), 8.60(\mathrm{~d}, \mathrm{~J}=1.4 \mathrm{~Hz}, 1 \mathrm{H}), 8.40(\mathrm{~s}, 2 \mathrm{H})$, $8.17(\mathrm{~s}, 1 \mathrm{H}), 7.98(\mathrm{~s}, 2 \mathrm{H}), 7.84(\mathrm{~s}, 2 \mathrm{H}), 7.63$ $(\mathrm{d}, J=28.8 \mathrm{~Hz}, 3 \mathrm{H}), 7.38(\mathrm{~d}, J=25.5 \mathrm{~Hz}, 3 \mathrm{H})$; ${ }^{13} \mathrm{C}$ NMR (76 MHz, DMSO- $\left.d_{6}\right) \delta 159.5,158.5$, 150.7, 141.2, 139.3, 138.7, 135.0, 133.9, 133.6, $132.2,131.5,130.6,130.4,128.7,128.4,128.3$, 127.0, 126.3, 124.0, 123.5, 120.3, 114.5, 113.0, 111.0 .

2-(2-Chlorophenyl)-N-[3-(3,4dichlorophenyl)-1H-indazol-5-yl]quinazolin4-amine hydrochloride 39. Yield $65 \% .{ }^{1} \mathrm{H}$ NMR (302 MHz, DMSO- $d_{6}$ ) $\delta 13.47$ (br s, $1 \mathrm{H}), 10.14$ (br s, 1H), 8.76 (s, 1H), 8.66 (d, $J$ $=8.1 \mathrm{~Hz}, 1 \mathrm{H}), 8.11(\mathrm{~s}, 1 \mathrm{H}), 7.86(\mathrm{~d}, J=7.0$ $\mathrm{Hz}, 2 \mathrm{H}), 7.72(\mathrm{~d}, J=7.3 \mathrm{~Hz}, 1 \mathrm{H}), 7.65(\mathrm{~d}, J$ $=7.2 \mathrm{~Hz}, 2 \mathrm{H}), 7.59(\mathrm{~d}, J=9.1 \mathrm{~Hz}, 2 \mathrm{H}), 7.50$ $(\mathrm{d}, J=7.7 \mathrm{~Hz}, 1 \mathrm{H}), 7.46-7.39(\mathrm{~m}, 2 \mathrm{H}), 7.37$ $(\mathrm{d}, J=7.3 \mathrm{~Hz}, 1 \mathrm{H}) ;{ }^{13} \mathrm{C}$ NMR $(76 \mathrm{MHz}$, DMSO- $\left.d_{6}\right) \delta 161.1,158.3,150.3,141.1,139.6$, 139.4, 134.9, 133.8, 133.6, 132.2, 131.9, 131.8, $131.4,130.4,130.3,128.3,128.3,127.3,126.9$, $126.7,123.9,123.5,120.2,114.2,113.3,111.1$.

$\mathrm{N}$-[3-(3,4-Dichlorophenyl)-1H-indazol-5yl]-6-methylquinazolin-4-amine hydrochloride 40. Yield $64 \% .{ }^{1} \mathrm{H}$ NMR (302 MHz, DMSO- $d_{6}$ ) $\delta 13.65$ (br s, 1H), 11.09 (br s, 1H), 8.70 (s, $1 \mathrm{H}), 8.63(\mathrm{~s}, 1 \mathrm{H}), 8.41(\mathrm{~s}, 1 \mathrm{H}), 8.09(\mathrm{~s}, 1 \mathrm{H})$, $7.92(\mathrm{~d}, J=7.8 \mathrm{~Hz}, 1 \mathrm{H}), 7.83-7.67(\mathrm{~m}, 4 \mathrm{H})$, $7.63(\mathrm{~d}, J=7.6 \mathrm{~Hz}, 1 \mathrm{H}), 2.48(\mathrm{~s}, 3 \mathrm{H}) ;{ }^{13} \mathrm{C}$ NMR (76 MHz, DMSO- $\left.d_{6}\right) \delta 159.2,151.8$, 141.1, 140.9, 140.1, 138.1, 136.8, 134.6, 132.1, $131.5,130.5,128.1,126.8,124.7,123.6,122.6$, 120.0, 115.4, 114.3, 111.2, 21.6.

3-(3,4-Dichlorophenyl)-N-(6phenylpyrimidin-4-yl)-1H-indazol-5-amine 41. Yield $80 \%$. ${ }^{1} \mathrm{H}$ NMR (400 MHz, DMSO- $d_{6}$ ) $\delta 13.65(\mathrm{~s}, 1 \mathrm{H}), 11.60(\mathrm{~s}, 1 \mathrm{H}), 8.84(\mathrm{~s}, 1 \mathrm{H})$, 
$8.41(\mathrm{~s}, 1 \mathrm{H}), 8.07(\mathrm{~s}, 1 \mathrm{H}), 8.00-7.85(\mathrm{~m}, 3 \mathrm{H})$, $7.73(\mathrm{~d}, J=8.2 \mathrm{~Hz}, 1 \mathrm{H}), 7.67(\mathrm{~s}, 1 \mathrm{H}), 7.63$ (d, $J=9.3 \mathrm{~Hz}, 1 \mathrm{H}), 7.58(\mathrm{~d}, J=6.8 \mathrm{~Hz}, 3 \mathrm{H}), 7.43$ $(\mathrm{s}, 1 \mathrm{H}) .{ }^{13} \mathrm{C}$ NMR (101 MHz, DMSO- $\left.d_{6}\right) \delta$ 162.0, 154.3, 154.2, 141.2, 139.8, 134.6, 132.4, $132.2,131.7,131.6,130.5,129.8,128.3,127.6$, 126.9, 122.9, 120.2, 113.3, 111.9, 104.3, 103.2.

N-[3-(3,4-Dichlorophenyl)-1H-indazol-5yl]-6,7-dimethoxy-2-(trifluoromethyl)quinazolin-4-amine 42. Yield $68 \%$. ${ }^{1} \mathrm{H}$ NMR (400 MHz, DMSO- $d_{6}$ ) $\delta 13.49$ (br s, $\left.1 \mathrm{H}\right), 9.99$ (br s, 1H), $8.72(\mathrm{~s}, 1 \mathrm{H}), 8.13(\mathrm{~s}, 1 \mathrm{H}), 7.97$ (d, $J=7.2 \mathrm{~Hz}, 2 \mathrm{H}), 7.75(\mathrm{~d}, J=8.7 \mathrm{~Hz}, 1 \mathrm{H}), 7.69$ $(\mathrm{d}, J=8.3 \mathrm{~Hz}, 1 \mathrm{H}), 7.64$ (d, $J=8.8 \mathrm{~Hz}, 1 \mathrm{H})$, 7.33 (s, 1H), $3.98(\mathrm{~s}, 3 \mathrm{H}), 3.93(\mathrm{~s}, 3 \mathrm{H}) .{ }^{13} \mathrm{C}$ NMR $\left(101 \mathrm{MHz}, \mathrm{DMSO}-d_{6}\right) \delta 157.5,155.3$, $150.7,147.5,146.3,141.0,139.4,134.9,133.4$, $132.2,131.3,130.4,128.1,126.7,123.6,122.0$, 120.1, 113.0, 111.1, 109.5, 108.3, 102.6, 56.9, 56.5 .

N-[3-(3,4-Dichlorophenyl)-1H-indazol-5yl]quinolin-4-amine hydrochloride 43. Yield $38 \%$. ${ }^{1} \mathrm{H}$ NMR (302 MHz, DMSO- $d_{6}$ ) $\delta 14.73$ (br s, 1H), 13.86 (br s, 1H), 11.26 (br s, 1H), $8.90(\mathrm{~d}, J=8.5 \mathrm{~Hz}, 1 \mathrm{H}), 8.43(\mathrm{~d}, J=6.7 \mathrm{~Hz}$, $1 \mathrm{H}), 8.21(\mathrm{~s}, 1 \mathrm{H}), 8.12(\mathrm{~s}, 1 \mathrm{H}), 8.09(\mathrm{~d}, J=$ $8.7 \mathrm{~Hz}, 1 \mathrm{H}), 7.99$ (t, $J=8.1 \mathrm{~Hz}, 2 \mathrm{H}), 7.79$ (d, $J=8.8 \mathrm{~Hz}, 1 \mathrm{H}), 7.75(\mathrm{~d}, J=7.8 \mathrm{~Hz}, 1 \mathrm{H}), 7.69$ (d, $J=8.4 \mathrm{~Hz}, 1 \mathrm{H}), 7.49$ (d, $J=9.0 \mathrm{~Hz}, 1 \mathrm{H})$, $6.69(\mathrm{~d}, J=6.9 \mathrm{~Hz}, 1 \mathrm{H}) ;{ }^{13} \mathrm{C} \mathrm{NMR}(76 \mathrm{MHz}$, DMSO- $\left.d_{6}\right) \delta 156.2,142.8,141.5,141.0,138.6$, $134.3,134.2,132.2,131.6,131.4,130.7,128.3$, $127.3,127.1,125.5,124.3,120.6,120.5,118.3$, $117.4,112.9,100.2$.

N-[3-(3,4-Dichlorophenyl)-1H-indazol-5yl]-2,3-dihydro-1H-cyclopenta[b]quinolin9-amine hydrochloride 44. Yield $84 \%$. ${ }^{1} \mathrm{H}$ NMR (400 MHz, DMSO-d $\left.)_{6}\right) \delta 15.42$ (br s, 1H), 13.63 (br s, 1H), 10.73 (s, 1H), 8.72 (d,
$J=8.5 \mathrm{~Hz}, 1 \mathrm{H}), 8.18(\mathrm{~d}, J=8.7 \mathrm{~Hz}, 1 \mathrm{H}), 8.13$ $(\mathrm{d}, J=1.9 \mathrm{~Hz}, 1 \mathrm{H}), 7.94(\mathrm{dd}, J=10.5,1.9 \mathrm{~Hz}$, 2H), 7.92-7.86 (m, 1H), $7.68(\mathrm{~d}, J=8.5 \mathrm{~Hz}$, 2H), $7.63(\mathrm{~d}, J=8.4 \mathrm{~Hz}, 1 \mathrm{H}), 7.37$ (dd, $J=$ $8.9,1.5 \mathrm{~Hz}, 1 \mathrm{H}), 3.19(\mathrm{t}, J=7.6 \mathrm{~Hz}, 2 \mathrm{H}), 2.20$ (t, $J=7.3 \mathrm{~Hz}, 2 \mathrm{H}), 1.97$ (p, $J=7.8 \mathrm{~Hz}, 2 \mathrm{H})$. ${ }^{13} \mathrm{C}$ NMR (101 MHz, DMSO- $\left.d_{6}\right) \delta 158.4$, $156.3,150.5,147.3,137.7,137.2,135.9,134.0$, $132.6,132.3,131.4,130.4,129.5,129.5,129.1$, $128.4,127.5,119.0,117.9,116.6,99.5$.

N-[3-(3,4-Dichlorophenyl)-1H-indazol-5yl]thieno[2,3-d]pyrimidin-4-amine hydrochloride 45. Yield $27 \%$. ${ }^{1} \mathrm{H}$ NMR (400 MHz, DMSO- $\left.d_{6}\right) \delta 10.81$ (br s, $\left.1 \mathrm{H}\right), 8.53(\mathrm{~s}, 1 \mathrm{H})$, $8.45(\mathrm{~d}, J=1.9 \mathrm{~Hz}, 1 \mathrm{H}), 8.15(\mathrm{~d}, J=1.8 \mathrm{~Hz}$, $1 \mathrm{H}), 8.11-8.06(\mathrm{~m}, 1 \mathrm{H}), 7.97$ (dd, $J=8.4,1.9$ $\mathrm{Hz}, 1 \mathrm{H}), 7.75$ (dd, $J=8.8,1.7 \mathrm{~Hz}, 1 \mathrm{H}), 7.69$ $(\mathrm{d}, J=5.9 \mathrm{~Hz}, 1 \mathrm{H}), 7.67(\mathrm{~d}, J=3.4 \mathrm{~Hz}, 1 \mathrm{H})$, $7.65(\mathrm{~d}, J=3.9 \mathrm{~Hz}, 1 \mathrm{H}) .{ }^{13} \mathrm{C} \mathrm{NMR}(101 \mathrm{MHz}$, DMSO- $\left.d_{6}\right) \delta 164.7,156.2,154.7,150.6,150.5$, $134.0,132.3,131.4,130.4,129.5,129.5,127.5$, 126.1, 121.0, 120.9, 120.5, 117.5, 113.6, 101.5.

N-[3-(3,4-Dichlorophenyl)-1H-indazol-5yl]-1-methyl-pyrazolo[3,4-d]pyrimidin4-amine hydrochloride 46. Yield $57 \%$. ${ }^{1} \mathrm{H}$ NMR (400 MHz, DMSO- $d_{6}$ ) $\delta 13.53$ (br s, $1 \mathrm{H}), 10.30$ (br s, 1H), 8.52 (s, 1H), 8.41 (s, $1 \mathrm{H}), 8.15(\mathrm{~d}, J=1.7 \mathrm{~Hz}, 1 \mathrm{H}), 7.97$ (dd, $J=$ 8.4, $1.7 \mathrm{~Hz}, 1 \mathrm{H}), 7.81(\mathrm{dd}, J=10.8,6.9 \mathrm{~Hz}$, 2H), $7.66(\mathrm{~d}, J=9.0 \mathrm{~Hz}, 1 \mathrm{H}), 7.58(\mathrm{t}, J=9.5$ $\mathrm{Hz}, 1 \mathrm{H}), 3.95$ (s, 3H). ${ }^{13} \mathrm{C}$ NMR (101 MHz, DMSO- $\left.d_{6}\right) \delta 158.0,155.7,151.5,150.5,148.4$, $134.0,132.3,131.4,130.6,130.4,129.5,129.5$, 129.4, 127.5, 118.9, 116.9, 101.6, 99.4, 33.4.

N-[3-(3,4-Dichlorophenyl)-1H-indazol-5yl]-1,3-dimethyl-pyrazolo[3,4-d]pyrimidin4-amine 47. Yield $48 \%$. ${ }^{1} \mathrm{H}$ NMR (400 MHz, DMSO- $\left.d_{6}\right) \delta 13.25$ (br s, $\left.1 \mathrm{H}\right), 8.69(\mathrm{~s}, 1 \mathrm{H})$, $8.32(\mathrm{~s}, 1 \mathrm{H}), 8.19(\mathrm{~s}, 1 \mathrm{H}), 8.16(\mathrm{~d}, J=1.9 \mathrm{~Hz}$, 
1H), $7.96(\mathrm{dd}, J=8.4,1.9 \mathrm{~Hz}, 1 \mathrm{H}), 7.92(\mathrm{~s}$, $1 \mathrm{H}), 7.68(\mathrm{dd}, J=8.8,1.5 \mathrm{~Hz}, 2 \mathrm{H}), 7.67$ (d, $J=8.4 \mathrm{~Hz}, 2 \mathrm{H}), 7.57(\mathrm{~d}, J=8.9 \mathrm{~Hz}, 1 \mathrm{H}), 3.91$ (s, 3H), $2.71(\mathrm{~s}, 3 \mathrm{H}) \cdot{ }^{13} \mathrm{C}$ NMR $(101 \mathrm{MHz}$, DMSO- $\left.d_{6}\right) \delta 156.1,155.5,154.4,140.8,140.3$, 139.8, 134.9, 133.4, 132.1, 131.5, 130.3, 128.1, $126.8,125.3,120.2,114.4,111.0,100.0,33.5$, 15.0.

3-Bromo-N-[3-(3,4-dichlorophenyl)- $1 \mathrm{H}$ indazol-5-yl]-1-methyl-pyrazolo[3,4-d]pyrimidin-4-amine 48. Yield $73 \%$. ${ }^{1} \mathrm{H}$ NMR (400 MHz, DMSO- $d_{6}$ ) $\delta 13.47$ (br s, $1 \mathrm{H}$ ), $10.21($ br s, $1 \mathrm{H}), 8.50(\mathrm{~s}, 1 \mathrm{H}), 8.42(\mathrm{~s}, 1 \mathrm{H})$, $8.17(\mathrm{~d}, J=1.8 \mathrm{~Hz}, 1 \mathrm{H}), 7.98(\mathrm{dd}, J=8.4$, $1.9 \mathrm{~Hz}, 1 \mathrm{H}), 7.89-7.74(\mathrm{~m}, 3 \mathrm{H}), 7.69$ (d, $J=$ $8.8 \mathrm{~Hz}, 1 \mathrm{H}), 3.96(\mathrm{~s}, 3 \mathrm{H}) .{ }^{13} \mathrm{C} \mathrm{NMR}(101 \mathrm{MHz}$, DMSO- $\left.d_{6}\right) \delta 156.6,154.3,152.2,150.5,149.1$, 134.0, 132.3, 131.4, 130.4, 129.5, 129.5, 129.4, 127.5, 119.8, 116.9, 113.6, 101.8, 100.3, 33.6.

$\mathrm{N}$-[3-(3,4-Dichlorophenyl)-1H-indazol-5yl]-1,6-dimethyl-pyrazolo[3,4-d]pyrimidin4-amine hydrochloride 49. Yield $62 \% .{ }^{1} \mathrm{H}$ NMR (400 MHz, DMSO- $d_{6}$ ) $\delta 13.57$ (br s, $1 \mathrm{H}), 10.45$ (br s, 1H), $8.93(\mathrm{~s}, 1 \mathrm{H}), 8.17$ (d, $J=1.8 \mathrm{~Hz}, 1 \mathrm{H}), 7.99(\mathrm{dd}, J=8.3,1.1 \mathrm{~Hz}, 1 \mathrm{H})$, $7.78(\mathrm{~d}, J=8.1 \mathrm{~Hz}, 1 \mathrm{H}), 7.75-7.62(\mathrm{~m}, 3 \mathrm{H})$, $3.92(\mathrm{~s}, 3 \mathrm{H}), 2.59(\mathrm{~s}, 3 \mathrm{H}) .{ }^{13} \mathrm{C}$ NMR $(101 \mathrm{MHz}$, DMSO- $\left.d_{6}\right) \delta 159.2,155.1,153.8,150.5,147.6$, 134.0, 132.3, 131.4, 130.6, 130.4, 129.5, 129.5,

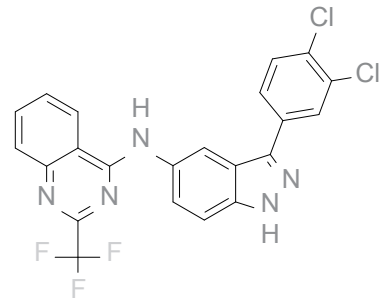

Compound 1

$\mathrm{IC}_{50}=0.007 \mu \mathrm{M}$

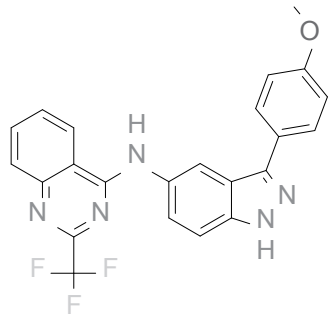

Compound 2 $\mathrm{IC}_{50}=0.016 \mu \mathrm{M}$
129.4, 127.5, 118.9, 116.9, 100.6, 99.4, 33.4, 25.7.

\section{LogP calculation}

LogP values were calculated using Marvin Sketch 19.12 (https://www.chemaxon.com).

\section{Results and Discussion}

Earlier we have found new promising CK2 inhibitors among the 4-quinazoline derivatives of 5-amino-3-arylindazoles 1-3 [29]. The inhibitory activity of these compounds against CK2 was tested in in vitro assay with radiolabeled ATP [30]. They have shown $\mathrm{IC}_{50}$ values in a range $0.007-0.1 \mu \mathrm{M}$ [29]. The structures and inhibitory activity of 1-3 are represented in Figure 2.

Besides the high $\mathrm{IC}_{50}$ values all three compounds 1-3 also have a significant disadvantage. They have too high lipophilicity $(\log \mathrm{P}>5)$ and as a result - poor water solubility. We have tried to modify chemical structure to overcome this disadvantage. We have selected the 3,4-dichlorophenyl substituent in the 3-position as a substituent of the most active compound $\mathbf{3}$ and left it unchanged. Meanwhile, we have focused on varying of available heterocyclic residues attached to the 5-amino-group. The 5-amino3-(3,4-dichlorophenyl)- $1 H$-indazole 6 interme-

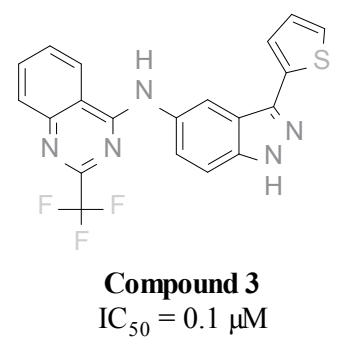

Fig. 2. Chemical structure of the 5-(4-quinazolylamino)-3-arylindazole derivatives 1-3. 
4
5

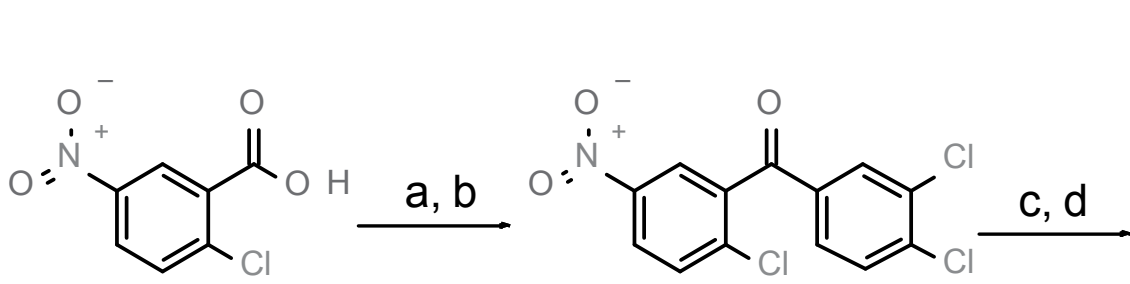

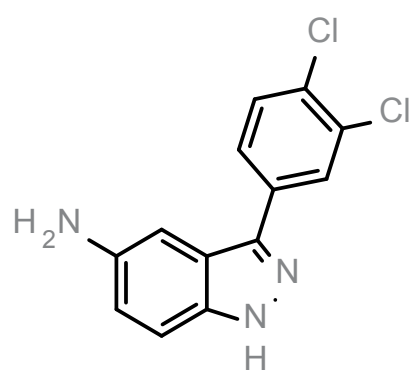

6

Scheme 1. The synthesis [of] 5-amino-3-(3,4-dichlorophenyl)-1H-indazole. a: $\mathrm{SOCl}_{2} ;$ b: 1,2-dichlorobenzene, $\mathrm{AlCl}_{3}$; c: $\mathrm{N}_{2} \mathrm{H}_{4} \times \mathrm{H}_{2} \mathrm{O}, \mathrm{DMF} ; \mathrm{d}: \mathrm{N}_{2} \mathrm{H}_{4} \times \mathrm{H}_{2} \mathrm{O}$, NiRe, iPrOH, DMF.

diate has been synthesized following Scheme 1 .

2-Chloro-5-nitrobenzoic acid 4 was converted into acid chloride and immediately used for Friedel-Crafts acylation of 1,2-dichlorobenzene. The heating of obtained ketone 5 with hydrazine hydrate in dimethylformamide caused chlorine substitution and indazole ring closure. The reduction of nitro-group with hydrazine hydrate in the presence of Raney nickel gave 5-amino-3-(3,4-dichlorophenyl)$1 H$-indazole 6.

Following starting heteroaromatic derivatives: (3H-quinazolin-4-one 8 [31], 2-isopropyl-3Hquinazolin-4-one 9 [32], 2-trifluoromethyl-3Hquinazolin-4-one [33], 2-phenyl-3H-quinazolin4-one 10, 2-(2-chlorophenyl)-3H-quinazolin-4one 11 [34], 6-methyl-3H-quinazolin-4-one 12 [31], 6-phenyl-3H-pyrimidin-4-one 13 [35], 6,7-dimethoxy-2-(trifluoromethyl)-3H-quinazolin-4-one 14 [36], 1H-quinolin-4-one 15 [37], 1,2,3,4-tetrahydrocyclopenta[b]quinolin-9-one 16 [38], 1H-thieno[2,3-d]pyrimidin-4-one 17 [39], 1-methyl-7H-pyrazolo[3,4-d]pyrimidin4-one 18, 1,3-dimethyl-7H-pyrazolo[3,4-d]pyrimidin-4-one 19 [40], 3-bromo-1-methyl-7Hpyrazolo[3,4-d]pyrimidin-4-one 20 [41], 1,6-di-
methyl-7H-pyrazolo[3,4-d]pyrimidin-4-one 21 [42]) were synthesized according to the known procedures. Treatment of these compounds with phosphorus oxychloride has converted them into the reactive chloro-derivatives 22-35 (Scheme 2). These intermediates, having been used in reaction with 5-amino-3-(3,4-dichlorophenyl)- $1 H$ indazole immediately after isolation, gave 5-Heterylamino- $1 H$-Indazoles 36-49. The substitution of $\mathrm{Cl}$ by 6 was carried out under refluxing of equimolar amounts of initial components in DMF giving only exocyclic nitrogen derivatives 36-49. The products 36-49 were precipitated as solid $\mathrm{HCl}$ salts. If precipitation did not take place, the products were converted to the base form by addition of triethylamine to the reaction mixture and dilution with water.

All novel synthesized compounds were tested for in vitro inhibitory activity towards CK2. Results of this testing as well as SAR and docking studies will be described in the separate article in the next issue of this journal.

\section{Conclusions}

Thus, following the previously used synthetic route, a series of 14 novel 5-hetarylamino-3-arylindazole derivatives was synthesized. 


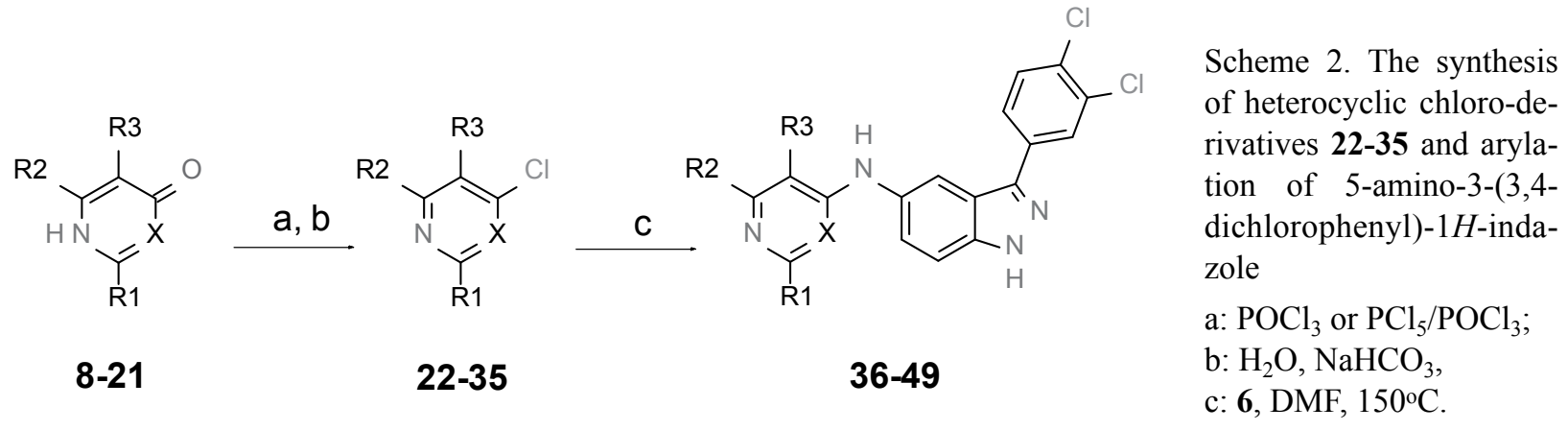

Besides additional quinazoline derivatives the quinoline and thieno[2,3-d]pyrimidine derivatives of similar structure but different polarity were obtained. Also a series of 1-methylpyrazolo[3,4-d]pyrimidine derivatives with decreased lipophilicity was synthesized.

\section{REFERENCES:}

1. Burnett $G$, Kennedy EP. The enzymatic phosphorylation of proteins. $J$ Biol Chem. 1954; 211(2):969-80.

2. Bian $Y$, Ye $M$, Wang $C$, Cheng $K$, Song $C$, Dong $M$, Pan Y, Qin H, Zou H. Global screening of CK2 kinase substrates by an integrated phosphoproteomics workflow. Sci Rep. 2013; 3:3460.

3. Hanks SK, Hunter T. The eukaryotic protein kinase superfamily: kinase (catalytic) domain structure and classification 1. FASEB J. 1995; 9(8):576-96.

4. Wilson LK, Dhillon N, Thorner J, Martin GS. Casein kinase II catalyzes tyrosine phosphorylation of the yeast nucleolar immunophilin Fpr3. J Biol Chem. 1997; 272(20):12961-7.

5. Faust M, Montenarh $M$. Subcellular localization of protein kinase CK2: A key to its function? Cell Tissue Res. 2000; 301(3):329-40.

6. Litchfield $D W$. Protein kinase CK2: Structure, regulation and role in cellular decisions of life and death. Biochem J. 2003; 369(Pt1):1-15.

7. Guerra B, Issinger $O-G$. Protein Kinase CK2 in Human Diseases. Curr Med Chem. 2008; 15(19): 1870-86.
8. Zandomeni R, Carrera Zandomeni M, Shugar D, Weinmann $R$. Casein kinase type II is involved in the inhibition by 5,6-dichloro-1- $\beta$-D-ribo-furanosylben-zimidazole of specific RNA polymerase II transcription. J Biol Chem. 1986; 261(7):3414-9.

9. Cozza G, Pinna LA, Moro S. Protein kinase CK2 inhibitors: A patent review. Expert Opin Ther Pat. 2012; 22(9):1081-97.

10. Prykhod'ko AO, Dubinina GG, Yarmoluk SM. Inhibitors of protein kinase CK2. Ukr Bioorg Acta. 2004; 1:39-48.

11. Chilin A, Battistutta R, Bortolato A, Cozza G, Zanatta S, Poletto G, Mazzorana M, Zagotto G, Uriarte E, Guiotto A, Pinna LA, Meggio F, Moro S. Coumarin as attractive Casein Kinase 2 (CK2) inhibitor scaffold: An integrate approach to elucidate the putative binding motif and explain structure-activity relationships. J Med Chem. 2008; 51(4):752-9.

12. Battistutta R, Mazzorana M, Sarno S, Kazimierczuk Z, Zanotti G, Pinna LA. Inspecting the structureactivity relationship of protein kinase CK2 inhibitors derived from tetrabromo-benzimidazole. Chem Biol. 2005;12:1211-1219.

13. Papinutto E, Ranchio A, Lolli G, Pinna LA, Battistutta $R$. Structural and functional analysis of the flexible regions of the catalytic $\alpha$-subunit of protein kinase CK2. J Struct Biol. 2012; 177(2):382-91.

14. Sarno S, De Moliner E, Ruzzene M, Pagano M, Battistutta R, Bain J, Fabbio D, Schoepfer J, Elliott M, Furet P, Meggio F, Zanotti G, Pinna LA. Biochemical and three-dimensional-structural study of the specific inhibition of protein kinase CK2 by 
[5-oxo-5,6-dihydroindolo-(1,2-a)quinazolin-7-yl] acetic acid (IQA). Biochem J. 2003; 374(Pt 3):63946.

15. Golub AG, Bdzhola VG, Briukhovetska NV, Balanda AO, Kukharenko OP, Kotey IM, Ostrynska OV, Yarmoluk SM. Synthesis and biological evaluation of substituted (thieno[2,3-d]pyrimidin-4-ylthio)carboxylic acids as inhibitors of human protein kinase CK2. Eur J Med Chem. 2011; 46(3):870-6.

16. Ostrynska OV, Balanda AO, Bdzhola VG, Golub $A G$, Kotey IM, Kukharenko OP, Gryshchenko AA, Briukhovetska NV, Yarmoluk SM. Design and synthesis of novel protein kinase CK2 inhibitors on the base of 4-aminothieno[2,3-d]pyrimidines. Eur J Med Chem. 2016; 115:148-60.

17. Golub AG, Yakovenko OY, Prykhod'ko AO, Lukashov SS, Bdzhola VG, Yarmoluk SM. Evaluation of 4,5,6,7-tetrahalogeno-1H-isoindole-1,3(2H)-diones as inhibitors of human protein kinase CK2. Biochim Biophys Acta - Proteins Proteomics. 2008; 1784(1):143-9.

18. Lolli G, Cozza G, Mazzorana M, Tibaldi E, Cesaro L, Donella-Deana A, Meggio F, Venerando A, Franchin C, Sarno S, Battistutta R, Pinna LA. Inhibition of protein kinase CK2 by flavonoids and tyrphostins. A structural insight. Biochemistry. 2012; 51(31):6097-107.

19. Golub AG, Bdzhola VG, Ostrynska OV, Kyshenia IV, Sapelkin VM, Prykhod'ko AO, Kukharenko OP, Yarmoluk SM. Discovery and characterization of synthetic 4'-hydroxyflavones - New CK2 inhibitors from flavone family. Bioorganic Med Chem. 2013; 21(21):6681-9.

20. Golub AG, Bdzhola VG, Kyshenia YV, Sapelkin VM, Prykhod'ko AO, Kukharenko OP, Ostrynska OV, Yarmoluk SM. Structure-based discovery of novel flavonol inhibitors of human protein kinase CK2. Mol Cell Biochem. 2011; 356(1-2):107-15.

21. Protopopov MV, Volynets GP, Starosyla SA, Vdovin VS, Lukashov SS, Bilokin YV, Bdzhola VG, Yarmoluk SM. Identification of 1,3-thiazole-5-carboxylic Acid Derivatives as Inhibitors of Protein Kinase CK2. Curr Enzym Inhib. 2017; 14(2):152-9.

22. Golub AG, Yakovenko OY, Bdzhola VG, Sapelkin VM, Zien P, Yarmoluk SM. Evaluation of 3-car-
boxy-4(1H)-quinolones as inhibitors of human protein kinase CK2. J Med Chem. 2006; 49(22):644350.

23. Protopopov MV, Vdovin VS, Starosyla SA, Borysenko IP, Prykhod'ko AO, Lukashov SS, Bilokin YV, Bdzhola VG, Yarmoluk SM. Flavone inspired discovery of benzylidenebenzofuran-3(2H)-ones (aurones) as potent inhibitors of human protein kinase CK2. Bioorg Chem. 2020; 102:104062.

24. Nakanishi I, Murata K, Nagata N, Kurono M, Kinoshita T, Yasue M, Miyazaki T, Takei Y, Nakamura S, Sakurai A, Iwamoto N, Nishiwaki K, Nakaniwa T, Sekuguchi Y, Hirasawa A, Tsujimoto G, Kitaura K. Identification of protein kinase CK2 inhibitors using solvent dipole ordering virtual screening. Eur J Med Chem. 2015; 96:396-404.

25. Ferguson AD, Sheth PR, Basso AD, Paliwal S, Gray K, Fischmann TO, Le HV. Structural basis of CX-4945 binding to human protein kinase CK2. FEBS Lett. 2011; 585(1):104-10.

26. Battistutta R, Cozza G, Pierre F, Papinutto E, Lolli G, Sarno S, O'Brien SE, Siddiqui-Jain A, Haddach M, An-deres K, Ryckman DM, Meggio F, Pinna LA. Unprecedented selectivity and structural determinants of a new class of protein kinase CK2 inhibitors in clinical trials for the treatment of cancer. Biochemistry. 2011; 50(39):8478-88.

27. Wan $Y$, He S, Li W, Tang Z. Indazole Derivatives: Promising Anti-tumor Agents. Anticancer Agents Med Chem. 2018; 18(9):1228-34.

28. Lukashov SS. Amino-3-arylindazole as template for Ser/Thr and Tyr kinase inhibitors revealing. $U k r$ Bioorganica Acta. 2009; 7:57-61.

29. Lukashov SS, Kukharenko OP, Bdzhola VG, Yarmoluk SM. Synthesis of 5-amino-3-arylindazole derivatives and study of their in vitro activity towards Ser/Thr and Tyr protein kinases. V International Conference "Chemistry of Nitrogen Containing Heterocycles», Kharkiv. 2009:O.21.

30. Hastie CJ, McLauchlan HJ, Cohen P. Assay of protein kinases using radiolabeled ATP: a protocol. Nat Protoc. 2006; 1(2):968-71.

31. Jones TR, Thornton TJ, Flinn A, Jackman AL, Newell DR, Calvert AH. Quinazoline Antifolates Inhibiting Thymidylate Synthase: 2-Desamino Deriva- 
The synthesis of 5-hetarylamino-3-aryl-1H-indazoles as inhibitors of protein kinase CK2

tives with Enhanced Solubility and Potency. $J$ Med Chem. 1989; 32(4):847-52.

32. Segarra V, Crespo MI, Pujol F, Beleta J, Domenech T, Miralpeix M, Palacios JM, Castro A, Martinez $A$. Phosphodiesterase inhibitory properties of losartan. Design and synthesis of new lead compounds. Bioorganic Med Chem Lett. 1998; 8(5):505-10.

33. Atkinson RS, Coogan MP, Cornell CL. Aziridination of alkenes using 3-acetoxyamino-2-trifluoromethylquinazolin-4(3H)-one. J Chem Soc - Perkin Trans 1. 1996(2):157-66.

34. Zentmyer DT, Wagner EC. The so-called acylanthranils (3,1,4-benzoxazones). I. Preparation; reactions with water, ammonia, and aniline; structure. $\mathrm{J} \mathrm{Org}$ Chem. 1949; 14(6):967-81.

35. Vanderhaeghe H, Claesen M. Pyrimidines II-Dérivés des acides pyrimidine-carboxyliques. Bull des Sociétés Chim Belges. 1957; 66:276-91.

36. Schwan G, Barbar Asskar G, Höfgen N, Kubicova L, Funke U, Egerland U, Zahn M, Nieber K, Scheunemann $M$, Sträter N, Brust P, Briel D. Fluorinecontaining 6,7-dialkoxybiaryl-based inhibitors for phosphodiesterase 10 A: Synthesis and in vitro evaluation of inhibitory potency, selectivity, and metabolism. Chem Med Chem. 2014; 9(7):1476-87.

37. Camps R. Von der Amidophenylpropiolsäure zur Kynurensäure und deren Verwandten. Berichte der Dtsch Chem Gesellschaft. 1901; 34:2703-18.

38. Brown RJ, Carver FWS, Hollingsworth BL. 506. The reaction of ethyl 2-oxocyclopentanecarboxylate with arylamines. Part II. The preparation of 2,3 -dihydro- $\beta$-quinindones $(2,3,4,9$-tetrahydro9-oxo-1H-cyclopenta[b]quinolines). $J$ Chem Soc. 1962:2624-6.

39. Yang $W$, Li L, Ji X, Wu X, Su M, Sheng L, Zang Y, Li J, Liu H. Design, synthesis and biological evaluation of 4-anilinothieno[2,3-d]pyrimidine-based hydroxamic acid derivatives as novel histone deacetylase inhibitors. Bioorg Med Chem. 2014; 22(21):6146-55.

40. Wahab Khan M, Uddin MK, Ali M, Rahman MS, Rashid MA, Chowdhury R. A convenient synthesis of new annelated pyrimidines and their biological importance. J Heterocycl Chem. 2014; 51(S1):E216E221.
41. Leonova TS, Babushkina TA, Yashunskii VG. Bromination of 4-oxopyrazolo[3,4-d]pyrimidines. Chem Heterocycl Compd. 1978; 14:326-31.

42. Morriello GJ, Chang L, Forster A, Berger R, Nanda $K K$, Shipe $W D$. Substituted pyrazolo/imidazolo bicyclic compounds as PDE2 inhibitors. 2016; WO2016209749.

\section{Синтез 5-гетариламіно-3-арил-1Н-індазолів як інгібіторів протеїнкінази СК2}

М. В. Протопопов, В. С. Вдовін, С. С. Лукашов, О. В. Остринська, І. П. Борисенко, О. В. Боровиков, С. А. Старосила, Я. В. Білокінь, О. П. Кухаренко, В. Г. Бджола, С. М. Ярмолюк

Мета. В продовження пошуку нових інгібіторів протеїнкінази СК2 грунтуючись на раніше виявленій нами інгібуючій активності похідних 5-(4-хіназоліламіно)3 -ариліндазолу було виконано синтез нових азотвмісних гетероциклічних похідних 5-аміно-3-арил-індазолу. Методи. Органічний синтез, спектроскопія ЯМР. Результати. Було синтезовано низку 4-хлорохіназолінів, 4-хлорохінолінів, 4-хлоропіразоло[3,4-d]піримідинів та 4-хлоротієно[2,3-d]піримідин. Взаємодією цих інтермедіатів з 5-аміно-3-(3,4-дихлорфеніл)-індазолом було одержано 14 нових гетероциклічних похідних 5-аміно-3-(3,4-дихлорфеніл)-індазолу. Висновки. Крім нових похідних хіназолину, було отримано похідні хіноліну та тієно[2,3-d]піримідину близькі за структурою, але відмінні за полярністю. Також було синтезовано низку 1-метилпіразоло[3,4-d]піримідинових похідних зі зниженою ліпофільністю.

К л юч о в і с л о в а: синтез, індазол, хіназолін, тієно[2,3-d]піримідин, піразоло[3,4-d]піримідин, інгібітор протеїнкінази СК2

\section{Синтез 5-гетариламино-3-арил-1Н-индазолов в качестве ингибиторов протеинкиназы СК2}

М. В. Протопопов, В. С. Вдовин, С. С. Лукашов, О. В. Остринская, І. П. Борисенко, О. В. Боровиков, С.А. Старосила, Я.В. Белоконь, О. П. Кухаренко, В. Г. Бджола, С. М. Ярмолюк

Цель. В продолжение поиска новых ингибиторов протеинкиназы CK2 опираясь на ранее выявленную нами 
ингибирующую активность производных 5-(4-хиназолиламино)-3-арилидазола были синтезированы новые азотсодержащие гетероциклические производные 5-амино-3-арил-индазола. Методы. Органический синтез, спектроскопия ЯМР. Результаты. Была синтезирована серия 4-хлорхиназолинов, 4-хлорхинолинов, 4-хлорпиразоло[3,4-d]пиримидинов и 4-хлортиено[2,3-d]пиримидин. Взаимодействием этих интермедиатов 3 5-амино-3-(3,4-дихлорфенил)-индазолом было получено 14 новых гетероциклических производных 5-амино-3-(3,4-дихлорфенил)-индазола. Выводы. Помимо новых производных хиназолина, были получены производные хинолина и тиено[2,3-d]пиримидина близкие по структуре, но другой полярности. Также была синтезирована серия 1-метилпиразоло[3,4-d]пиримидиновых производных с понижной липофильностью.

Кл юче вы е сл ов а: синтез, индазол, хиназолин, тиено[2,3-d]пиримидин, пиразоло[3,4-d]пиримидин, ингибитор протеинкиназы CK2

Received 05.10.2020 